\title{
Propuesta de acciones turísticas para el Sur de la Isla de la Juventud.
}

\author{
Touristic actions propose for the South of the Isle of Youth.
}

Daniel Crispín Rodríguez. ${ }^{1}$ Adriel Malvarez Cuello. ${ }^{2}$, Celia Amy Espinosa González. ${ }^{3}$, Celia \& Velasteguí López Efraín. ${ }^{4}$

\begin{abstract}
.
The development of a sustainable tourism activity has vital importance for any destination. But this management must be applied to take advantage of its resources and attractions. It's not recommendable to make an abusive use of the resources, it's necessary to develop the potential they have, so that tourism is developed that benefits the territory where it is carried out. The South Zone of the Isle of Youth, is a site of immense natural wealth that is not exempt from this problem; therefore, the objective of this study was to design a group of touristic actions to take advantage of the resources of the South of the Island. The greatest contribution of this research has been the elaboration of an action plan, in order to analyze the economic possibilities that this type of activities can report to the area, preserving its character of sustainable economy without compromising the environment for future generations, so that they can enjoy and admire the beauty of existing natural resources.
\end{abstract}

Keywords: Actions, Sustainable Development, Tourism, Nature, Ecotourism.

\section{Resumen.}

El desarrollo de una actividad turística sostenible es de vital importancia para cualquier destino. Pero se debe aplicar una correcta gestión para aprovechar sus recursos y atractivos. No se debe hacer un uso abusivo de sus recursos, tampoco se debe desaprovechar las potencialidades que estos tienen, para que se desarrolle un turismo que beneficie al territorio donde se realiza. La Zona Sur del Municipio Especial Isla de la Juventud, es un sitio de inmensa riqueza natural que no queda exento de esta problemática; por lo que el objetivo de este estudio fue diseñar un plan de acciones turísticas para el aprovechamiento de los recursos del Sur de la Isla. La mayor aportación de esta

${ }^{1}$ Universidad de la Habana, Facultad de Turismo, La Habana, Cuba, danicr97@yahoo.com

${ }^{2}$ Universidad de la Habana, Facultad de Turismo, La Habana, Cuba, adrielmalva2017@gmail.com

${ }^{3}$ Universidad de la Habana, Facultad de Comunicación, La Habana, Cuba, celiamy@nauta.cu

${ }^{4}$ Ciencia Digital Editorial, Ambato Ecuador, luisefrainvelastegui@cienciadigital.org 
investigación ha sido la elaboración de un plan de acciones, a fin de poder analizar las posibilidades económicas que este tipo de actividades pueden reportar a la zona, conservando su carácter de economía sostenible sin que se comprometa el medioambiente para las futuras generaciones, de manera que puedan disfrutar y admirar la belleza de los recursos naturales existentes.

Palabras claves: Acciones, Desarrollo Sostenible, Turismo, Naturaleza, Ecoturismo.

\section{Introducción}

La creciente demanda de los turistas por visitar destinos exclusivos y especialmente áreas protegidas y parques nacionales en todo el mundo genera una preocupación global en los propietarios y administradores de estos sitios, por la urgente necesidad de generar fondos económicos y recursos humanos capacitados; que le permitan mantener la integridad de sus áreas. Según datos publicados por la Organización Mundial del Turismo (OMT) en (Panorama OMT del Turismo Internacional. Edición 2015) "La región de las Américas (+8\%) registró el mayor crecimiento relativo de todas las regiones del mundo en 2014, recibiendo 13 millones más de turistas internacionales, y elevando a 181 millones el total de llegadas...".

La importancia del turismo recreativo en los últimos años ha ido incrementándose hasta convertirse en muchos países, en uno de los factores más importantes de su impacto económico, entendiendo como tal: "La medida de los beneficios y, también, de los costes económicos generados por el desarrollo de esta actividad" (Picornell, 2015).

El ecoturismo es una modalidad que se encuentra en auge en la actualidad y que comienza a ocupar lugares importantes en varios destinos del mundo, siendo el desarrollo de esta una manera efectiva de aprovechar los espacios forestales.

En Cuba existen zonas donde se han dado importantes pasos en el aprovechamiento de los espacios naturales con fines ecoturísticos turísticos; como por ejemplo en el Valle de Viñales en Pinar del Río, la Ciénaga de Zapata en Matanzas, algunas zonas de la Sierra Maestra y algunos cayos en Ciego de Ávila y Villa Clara. (Crispin, 2017).

Para desarrollar esta investigación, se tomó como referencia las cinco regiones naturales en las que en 1967 se dividió la Isla de la Juventud, Cuba y escogiéndose para realizar este estudio el Área Protegida de Recursos Manejados Sur de la Isla de la Juventud.

El Sur, es una zona turística que se considera en la actualidad un verdadero enigma para el mundo entero. Asociada a leyendas de corsarios y piratas, y con una flora y fauna espectacular ha visto el desarrollo del turismo, desde una posición de observador pasivo, muy alejada de los grandes resultados que exhiben otros destinos con similares potencialidades.

El alto valor natural, cultural e histórico de los lugares que componen esta zona la dota de grandes potencialidades para el desarrollo del turismo. Actualmente esas potencialidades no se aprovechan 
de la manera adecuada puesto que no se cuenta con la infraestructura necesaria para realizar la actividad turística a plenitud.

Pese a esto; según (Álvarez, 2018), el Sur recibió en 2017 un total de 55 embarcaciones de las cuales 3 fueron cruceros llegando por esa vía un total de aproximadamente 5000 visitantes en el Parque Nacional Marino de Punta Francés. Es frecuente además la llegada de yates con excursionistas (procedentes de Cayo Largo principalmente) a Punta del Este. Por la vía terrestre llegaron un total de 176 excursionistas extranjeros.

Estos datos indican que el principal desarrollo de la actividad turística de la zona se ha llevado a cabo a través de la náutica y el Crucerismo, aunque la zona posee un alto potencial para la realización de modalidades como el Ecoturismo, Turismo de Aventuras, Turismo Deportivo, Turismo Cultural y Turismo Científico, lo cual demuestra que el desarrollo del turismo en la zona es todavía insuficiente.

El bajo desarrollo del turismo en este territorio del Municipio Especial es una problemática importante a resolver por parte de las autoridades locales. Es por ello que, para dar solución a la misma, en la presente investigación se trazaron los siguientes objetivos:

\section{Objetivo General.}

Diseñar un plan de acciones turísticas para el aprovechamiento de los recursos del Sur de la Isla.

\section{Objetivos Específicos.}

1. Realizar una caracterización físico-geográfica y socio-económica del Sur de la Isla de la Juventud.

2. Diagnosticar la situación turística de la Zona Sur de la Isla de la Juventud.

3. Determinar la capacidad de carga de uso turístico de los sitios de estudio.

4. Proponer acciones para el aprovechamiento turístico de los sitios de estudio.

\section{Metodología.}

Para el desarrollo de la investigación fueron utilizados los siguientes métodos del nivel teórico:

- Análisis documental: Se utilizó durante toda la investigación para el marco teórico conceptual y durante el diagnóstico para constatar en los documentos normativos, tanto nacionales como internacionales, el estado de la problemática estudiada.

- Histórico - lógico: La utilización de este método permitió establecer la necesaria correspondencia entre los elementos de los métodos históricos y lógicos, con el fin de analizar la evolución histórica de los conceptos empleados en la investigación.

- Comparativo: Permitió valorar mediante la comparación las analogías y diferencias existentes entre los diferentes sitios de estudios, referente a sus valores naturales e históricos culturales. 
Se utilizaron los siguientes métodos del nivel empíricos:

Entrevista: Fue aplicada al Dr. C. Douglas Crispín Castellanos, Profesor Titular de la Facultad de Cultura Física de la Universidad Jesús Montané Oropesa de la Isla de la Juventud, y especialista en ecoturismo; y al MsC. Luis Felipe Álvarez Guerra, Especialista en Comercialización de la Agencia ECOTUR en la Isla de la Juventud; para la obtención de información acerca de los recursos y atractivos turísticos del Sur de la Isla de la Juventud y sus potencialidades para el desarrollo de actividades turísticas.

\section{Desarrollo.}

Capítulo I. Fundamentos Teóricos Conceptuales.

\section{Epígrafe 1. Medio Ambiente y Ecoturismo.}

El sector del turismo especializado en las incursiones en la naturaleza se denomina ecoturismo, logrando un gran auge en la actualidad; a la vez que se ha convertido en un verdadero reto para los propietarios y administradores de los parques nacionales y las áreas silvestres protegidas de todo el mundo mantener dichos espacios naturales mitigando los impactos negativos a sus ecosistemas, debido a que no todos los que se proclaman abanderados de este sector realmente cumplen con sus principios y se cubren en la imagen comercial de sus productos para lucrar con los recursos naturales que tienen en sus manos (Boo, E. 1990).

El ecoturismo es una modalidad a tener presente para el manejo de los recursos forestales y su aprovechamiento para el turismo, éste se define como "la ejecución de un viaje a áreas naturales que están relativamente sin disturbar o contaminar, con el objetivo específico de estudiar, admirar y gozar el panorama junto con sus plantas, animales silvestres y así mismo cualquier manifestación cultural (pasada y presente) que se encuentre en estas áreas...” (Ceballos- Lascurain, 1996).

La búsqueda de un equilibrio entre el disfrute del visitante y la prioridad de conservación es lo que caracteriza el concepto total y la filosofía del Ecoturismo en áreas protegidas. Los elementos distintivos del Ecoturismo son la no merma de los recursos, requiere una infraestructura mínima, lo practican personas que tienen un verdadero interés en las áreas visitadas, contribuye a los esfuerzos conservacionistas locales además del desarrollo social y económico. (UH / CIDA. 1996).

Para que la actividad turística realizada en la naturaleza sea considerada ecoturismo debe de realizarse con diferentes objetivos bien definidos los cuales son: contemplar, admirar, disfrutar, estudiar, respetar y conservar los recursos que la madre naturaleza ha puesto en nuestras manos para que las futuras generaciones puedan ellas mismas realizar dichos objetivos para lograr el verdadero desarrollo sostenible al cual aspiramos todos para nuestra sociedad. (Crispín, 2017).

Además, el ecoturismo no debe valorar solamente los recursos naturales de un área, debe de considerar también los valores históricos culturales que encierra la misma; porque sólo en su 
conjunto, es decir, unidos tanto los valores naturales e históricos culturales es que realmente se desarrolla el ecoturismo.

Capítulo 2. Diagnóstico de la Situación Turística del Sur de la Isla de la Juventud.

\section{Epígrafe 2.1 Análisis de la situación Turística.}

\subsubsection{Oferta}

Alojamiento

La oferta de alojamiento en el Sur de la Isla es muy limitada, prácticamente nula, debido a la ausencia de infraestructura de alojamiento. Solo se cuenta con algunas casas de rentas pertenecientes al sector no estatal en el Poblado de Cocodrilo las cuales no sobrepasan las 20 habitaciones, las mismas tienen un precio promedio de 3 cuc diario por habitación sin incluir los servicios de restauración. La mayoría de las habitaciones no poseen baño interior, ni climatización por lo que la calidad del alojamiento es baja.

\section{Restauración.}

Los servicios de restauración se comportan de manera similar a los de alojamiento, puesto que solo existe un restaurante y un bar - cafetería, ambos localizados en el Poblado de Cocodrilo, los cuales tienen una capacidad limitada, aproximadamente 20 pax cada uno con precios módicos y en Moneda Nacional. Las casas de rentas brindan estos servicios a un precio promedio de $40 \mathrm{MN}$ el almuerzo y la comida; y 15 MN el desayuno. Además, en el Parque nacional Marino de Punta Francés existen 2 ranchones para la oferta de bebidas y licores (principalmente de producción nacional) con un rango de precios de entre 1 y 15 cuc y un ranchón para la oferta de comida criolla con un rango de precios de 3 a 20 cuc.

\section{Deporte y Recreación.}

La oferta para la realización de actividades deportivas y de recreación es variada, y se cuenta con un alto potencial para su desarrollo. Las principales modalidades y actividades que se ofertan son:

1. Cabalgatas.

2. Jeep Safari.

3. Senderismo.

4. Caminatas.

5. Recorridos.

6. Observación de Aves.

7. Pesca.

8. Buceo.

9. Snorkeling

10. Kite Surf.

11. Caza.

12. Eventos Deportivos (Pesca de la Aguja, La Corrida del Pargo) 
13. Cicloturismo (existe potencial).

14. Rutas Ecoturísticas (existe potencial).

15. Rutas Culturales (existe potencial).

\subsubsection{Demanda.}

Los principales mercados emisores de turismo hacia el Sur de la Isla de la Juventud son Alemania, Reino Unido, Canadá y Estados Unidos. El mercado nacional tiene muy baja participación debido a los problemas existentes con las vías de acceso y el transporte. La mayoría de los visitantes que se reciben son excursionistas ya que la mayoría llegan a la zona en cruceros o embarcaciones y la capacidad de alojamiento es prácticamente nula dentro del Sur.

El Sur recibe visitantes de casi todas las edades, pero el rango predominante está entre los 50 - 70 años. Existe bastante paridad entre los sexos, pero el femenino es superior. La mayoría posee ingresos medios y sus motivaciones principales son el disfrute y contemplación del entorno, relajación e intercambio con el medio natural. Las modalidades más realizadas son, el Turismo de Sol y Playa y el Turismo Náutico. Los productos y actividades que más demandan son:

- Bebidas y licores.

- Comida Criolla.

- Artesanía Local.

- Snorkeling.

- Buceo.

Las principales insatisfacciones de la demanda están condicionadas por la falta y el mal estado de implementos para el descanso y la relajación en las playas, calidad de las bebidas, el déficit de medios de transporte y el mal estado de las vías de acceso terrestre.

\subsubsection{Infraestructura y Servicios.}

En el Sur de la isla no hay mucha infraestructura, no existen hoteles y solo se cuenta con un máximo de 20 habitaciones disponibles en casas de renta ubicadas en el Poblado de Cocodrilo. Existe un faro que facilita la navegación, hay una estación meteorológica ubicada en Punta del Este y una estación de biomasa forestal en Cocodrilo. Los servicios básicos con que se cuentan son:

- Salud: un consultorio médico en Cocodrilo con una ambulancia.

- Abasto de agua potable: Solo existe conductora en Cocodrilo, el resto de los sitios se abastecen mediante carros cisterna.

- Electricidad: La zona no está conectada a la red eléctrica del municipio. El poblado de Cocodrilo obtiene electricidad mediante plantas de diésel y una estación de biomasa forestal y en el Parque Nacional Marino de Punta Francés se emplean paneles solares. En otros sitios se emplean plantas de diésel. 
- Transporte: No existe infraestructura para el transporte, solo existe un ómnibus de servicio público que conecta a Cocodrilo con Nueva Gerona y realiza un viaje diario. No existen Agencias de Viaje ni de renta de autos.

Otros servicios que se prestan en el territorio son:

- Restauración: un restaurante, un bar cafetería, una panadería y 3 ranchones. Además, existen casas de renta que prestan servicios de restauración a sus clientes en el Poblado de Cocodrilo.

- Comercio: Existe una tienda perteneciente a la cadena TRD en el Poblado de Cocodrilo y algunos puntos ambulantes de venta de artesanías en Punta Francés.

- Recreación: Solo existe un Círculo Social ubicado en Cocodrilo.

Las vías de acceso son limitadas, no existe marina ni punto náutico; las embarcaciones de gran calado se mantienen cerca de la costa a golpe de máquina mientras que las embarcaciones de mediano y pequeño calado se amarran a un espigón sin echar anclas (por razones de conservación del fondo marino se prohíbe el uso de anclas). Las vías terrestres se encuentran en mal estado; para su evaluación se partió del siguiente criterio:

'Excelente es aquella carretera que no presenta ningún bache en todo su trayecto, está asfaltada y no presenta casi curvas; bueno cuando tiene baches aislados, está asfaltada y no presenta muchas curvas; regular es cuando presenta baches periódicos, no está asfaltada y presenta curvas con regularidad; mala es cuando tiene baches continuos, no está asfaltada y presenta muchas curvas. " (Crispín, 2016).

Como se puede apreciar la infraestructura con que cuenta el territorio es insuficiente para la realización de una actividad turística de calidad. Esto se debe a la mala política inversionista aplicada en el territorio, además de las limitaciones financieras del Municipio Especial y las cuestiones pertinentes a la conservación del área protegida las cuales limitan la construcción de edificaciones. La mayor parte de la infraestructura existente es rústica y no se encuentra en un buen estado de conservación por lo que es de vital importancia la restauración de la misma y la construcción de nuevas infraestructuras para lograr un mejor aprovechamiento de las potencialidades turísticas de la zona.

\subsubsection{Análisis de la Competencia.}

A nivel nacional el principal competidor del territorio es el Destino Cayo Largo del Sur por ser un destino consolidado de Sol y Playa, con un alto desarrollo del Turismo Náutico, por ser el destino más cercano y además de eso por pertenecer a la administración territorial del Municipio Especial Isla de la Juventud. Además de Cayo Largo otros territorios le hacen la competencia tales como la Ciénaga de Zapata, en Matanzas, y la Península de Guanacabibes en Pinar del Río, ambos constituyen áreas protegidas con alto valor para el Turismo de Naturaleza y el Ecoturismo y también poseen espacios litorales. En el ámbito internacional la mayor competencia la representan 
los destinos de América Central y el Caribe tales como, Cancún, Belice y Costa Rica, todos muy bien posicionados en el mercado de Sol y Playa, Turismo Náutico y/o de Naturaleza.

Puntos Fuertes:

Los puntos fuertes de los competidores que le otorgan ventajas son:

- Ser un Destino Turístico consolidado (Cayo Largo, Cancún, Belice y Costa Rica).

- Poseer una infraestructura turística superior.

- Imagen Consolidada y Estrategias de Marketing efectivas. (Cayo Largo, Cancún, Belice y Costa Rica).

- Mayor Comercialización.

- Mejor Accesibilidad.

Puntos Débiles:

Los puntos débiles de los competidores del territorio son:

- La mayoría de las construcciones están ubicadas sobre las dunas de la playa (Cayo Largo).

- Tratamiento de Residuales inapropiados (Cayo Largo).

- No tiene fuentes naturales de agua potable (Cayo Largo).

- No recibe visitas de cruceros (Cayo Largo y Ciénaga de Zapata).

- Presencia de Volcanes (Costa Rica).

- Presencia de especies peligrosas para la vida del hombre (Costa Rica, Belice, Cancún).

- Presencia del Narcotráfico (Costa Rica, Cancún y Belice).

Para lograr el éxito del territorio se debe aprender de las fortalezas de los rivales y sacar ventaja de sus debilidades. Además, se deben aprovechar los elementos que diferencian al territorio del resto los cuales son:

- Los fondos marinos del Parque Nacional de Punta Francés han sido catalogados por expertos italianos como los mejores conservados para la realización del buceo en el Caribe.

- Posee el Faro más alto de Latinoamérica (Faro de Carapachibey).

- Posee la cueva con más pictografías rupestres de todo el mundo a nivel de isla (Cueva No 1 de Punta del Este, con 212 pictografías).

- Posee la Primera Iglesia Luterana de Cuba (ubicada en Cocodrilo).

- Posee la mayor y mejor conservada población Uvas Caletas de Cuba.

\subsubsection{Identidad del Territorio.}

El territorio no tiene una imagen definida, y esto se debe en gran parte a la inadecuada gestión de la comercialización que se implementa en él. La agencia de viaje encargada de su comercialización en el territorio no dispone de la tecnología sufriente y no hace un buen uso de la que dispone para realizarla correctamente. La imagen del territorio llega generalmente a los clientes por medio de 
terceros (la agencia ECOTUR negocia directamente con las agencias de viajes y de cruceros) o mediante la propia agencia ECOTUR mediante la comunicación interpersonal. En la mayoría de los casos los terceros tampoco realizan una buena promoción de la imagen del territorio por lo que la imagen inducida no causa grandes expectativas en los clientes. La verdadera imagen del destino la perciben los visitantes dentro de él.

Pese a esto la imagen que transmite el Sur es la de un sitio apacible y misterioso, lleno de belleza natural y playas deliciosas. Pero esta no va a llegar a quienes no lo conocen si no se realiza una correcta gestión de su comercialización.

\subsubsection{Tendencias actuales.}

Según la situación actual y teniendo en cuenta las proyecciones futuras la llegada de cruceros tiende a mantenerse, mientras que el arribo de yates de mediano y bajo calado tiende al aumento. La demanda de otras actividades y de modalidades alternativas tales como el Ecoturismo, el Senderismo y Turismo de Aventuras se prevé que aumente, así como su participación dentro de la oferta del territorio. Debido a esto se experimentará un ligero mejoramiento de la infraestructura turística de la zona, pero la tendencia del crecimiento de proyectos inversionistas no es favorable. Pese a esto se pronostica que los visitantes anuales experimenten un crecimiento ligero.

Epígrafe 2.2. Caracterización físico geográfica.

El Área Protegida de Recursos Manejados Sur de la Isla de la Juventud se ubica como lo indica su nombre en la zona sur de la Isla de la Juventud (Segunda Isla por su extensión del Archipiélago Cubano, con $2199 \mathrm{~km} 2$ ). La altitud de la zona varía de $-6 \mathrm{~m}$ bajo el nivel medio del mar en las zonas sumergidas hasta los $30 \mathrm{msnm}$ (metros sobre el nivel del mar), en Cerro Caudal; con un promedio de altura de $3 \mathrm{msnm}$, se considera una llanura cársica. La acción de las aguas subterráneas ha originado la presencia de cuevas a lo largo de toda la costa.

Las profundidades de sus aguas raras veces superan los diez metros y su promedio es de siete metros. Estas aguas de escasa profundidad (asentados sobre porciones sumergidas de la región suroccidental de la plataforma insular y que abarcan un área de $34145 \mathrm{Km} 2$ ), condicionan grandes aportes de vapor de agua y tienden a elevar el calor latente y en consecuencia incrementan los regímenes diarios y anuales promedio de temperatura, precipitación y humedad.

A la altura de Punta del Este y hacia el oriente, la plataforma insular está festonada de hermosísimos cayos de gran interés ecológico, pues se ha demostrado que constituyen refugio de valiosas especies de flora y fauna. La extensión de sus playas y la finura de sus blancas arenas le confieren un elevado valor turístico. Desde este punto de vista, se integran como región al sur de la Isla.

La flora terrestre está representada por 556 especies de plantas superiores y 13 especies de helechos, con un endemismo de aproximadamente 105 especies, de ellas 3 son estrictas para el área. La flora marina presenta 42 especies de algas. 
La fauna se destaca por su diversidad y grado de endemismo. Se reportan 62 especies de aves residentes y 15 endémicas. Existen 20 especies de reptiles para el área. Se encuentran sitios de anidamiento de tortugas y poblaciones de iguanas, destacándose por su abundancia en los sitios El Guanal, el Parque Nacional Punta Francés, Playa Blanca y la Reserva Ecológica Punta del Este. Los mamíferos de la zona están representados por jutías y murciélagos; también es posible observar, en especial hacia las costas del oeste, al manatí. Son abundantes en el sur de la Isla los venados de cola blanca, especie introducida en el siglo pasado y naturalizada en el país.

El estimado de la fauna marina sobrepasa las 1500 especies entre corales (más de 45 especies), gorgonias, esponjas, crustáceos, quelonios, moluscos, gusanos de mar (31 especies solo en Punta del Este) y peces.

Esta zona es sitio RAMSAR y dentro de ella se encuentran otras áreas protegidas con categorías de manejos más restrictivas, como el Parque Nacional Punta Francés, la Reserva Ecológica Punta del Este, la Reserva Ecológica Ciénaga de Lanier y el Elemento Natural Destacado Pinar Calizo. (Flora y Fauna, 2015).

\section{Características Socioeconómicas.}

Al ser un área natural protegida, la población de la zona es limitada, solo existe un asentamiento poblacional, el poblado de Cocodrilo (antiguo Jacksonville) el cual tiene una población aproximada de 400 habitantes lo que representa aproximadamente el 0,47\% de la población total de la Isla de la Juventud. (ONEI, 2016).

Este poblado fue el primer asentamiento de caimaneros en la isla por lo cual su población se ha visto altamente influenciada por la cultura y las costumbres de estos antiguos habitantes pese a que los descendientes directos de estos son ya la minoría.

Sus habitantes viven de la pesca, la agricultura, producción de carbón, actividad forestal y al cuidado de la flora y fauna en áreas aledañas y el bosque. El acceso al pueblo se realiza fundamentalmente por tierra, aunque también puede lograrse por la cooperativa pesquera, a través de un muelle con condiciones mínimas por donde arriban las embarcaciones.

La actividad económica principal es la pesca, donde se destaca la captura de mariscos, principalmente la langosta y la pesca de esponjas marinas. El turismo también se desarrolla gracias a las condiciones y atractivos existentes en la zona.

Cuenta con una estación ecológica para la conservación de las especies autóctonas. Posee un centro de recría de tortugas que devuelven al mar cuando aumenta de talla. Unos kilómetros antes de llegar al poblado, en Cayo Potrero se ha construido un criadero de cocodrilo cubano.

Hoy día, el poblado está integrado por muy pocos de aquellos descendientes de caimaneros, ya que han sido absorbidos por la población autóctona. Lo que no queda duda, es que sus costumbres, 
quedaron muy arraigadas y han supervividos e influenciados entre la sociedad pinera. Dispone de muchas otras comodidades, que los antiguos fundadores jamás pensaron obtener.

La población actual dispone de electricidad generada por plantas de diésel, abasto de agua por conductoras (no totalmente restablecidas todas sus acometidas), escuelas pre-escolar y primaria, círculo infantil, panadería, emisora de radio local, bodega, oficina de correo y teléfono, círculo social, consultorio médico de familia con ambulancia, farmacia, estación de radio, servicios comerciales y gastronómicos.

Dejaron de construirse viviendas de madera por las de mampostería, que cuentan todas ellas con cocina de gas. Actualmente se trasladan casi a diario en un solo viaje de ida y vuelta en un viejo ómnibus hasta la ciudad de Nueva Gerona.

\subsubsection{Comparación de las áreas:}

El análisis comparativo de los tres sitios de estudio, es decir Punta del Este, Laguna Alvariño y Cocodrilo se desarrolló sobre la base de las características principales de su flora, fauna, paisaje y de los elementos antrópicos que se encuentran presentes en dichos sitios.

Tabla 1. Características generales de los sitios de estudio.

\begin{tabular}{cccc}
\hline $\begin{array}{c}\text { Variables. } \\
\text { Carretera. }\end{array}$ & $\begin{array}{c}\text { P. del Este. } \\
\text { Mal estado. }\end{array}$ & $\begin{array}{c}\text { Sitios. } \\
\text { Alvariño. } \\
\text { Regular estado. }\end{array}$ & $\begin{array}{c}\text { Cocodrilo. } \\
\text { Regular estado. }\end{array}$ \\
\hline Distancia desde punto 0 & 60 & 68 & 102 \\
(Km.) & & & \\
Paisaje. & Cuevas / playa / & Laguna / & Bosques / Playa. \\
& Bosques. & Bosques. & \\
Especies de la flora. & 24 & 11 & 1 \\
Formaciones vegetales. & 5 & 1 & 12 \\
Especies de aves. & 10 & 10 & 5 \\
Especies de reptiles. & 4 & 5 & 3 \\
Especie de mamíferos. & 2 & 3 & 4 \\
Moluscos gastrópodos. & 5 & 0 & 2 \\
Agentes nocivos. & 2 & 2 & 3 \\
Impactos. & 4 & 3 & Faro de \\
Valores históricos - & Pictografías / & Carapachibey & Caimaneros. \\
culturales. & cultura aborigen & Camiento de
\end{tabular}

Fuente: Elaboración propia.

Considerando todas las características, los 3 sitios poseen valores similares. En resumen, las áreas se compensan una a otra en sus valores de acceso y naturaleza e histórico - culturales.

Características de las especies atractivas. 
Para las especies de animales, aves y reptiles detectadas en los sitios de estudio y consideradas por el autor como atractivas (es decir, con aspectos atractivos para las actividades de recreación ecoturísticas), se ofrecen las características que las hacen ser así, y que deben ser resaltadas por los guías en sus explicaciones acerca de las especies que se observan. (anexo 1).

Epígrafe 2.3. Inventario de Recursos y Atractivos Turísticos.

Para el inventario de recursos se tuvo en cuenta los lugares señalados por el Dr. C. Douglas Crispín Castellanos, Profesor Titular de la Facultad de Cultura Física de la Universidad Jesús Montané Oropesa de la Isla de la Juventud, y especialista en ecoturismo en la entrevista realizada de los cuales se hizo una selección de los que se consideraron más significativos y adecuados para el desarrollo de la presente investigación. Por poseer un alto valor natural, cultural e histórico, por su carácter atrayente, estado conservatorio y por poseer al menos a pequeña escala infraestructura que facilite el desarrollo de actividades turísticas fueron escogidos los siguientes recursos y/o atractivos turísticos del Sur de la Isla de la Juventud para realizar el inventario de recursos y atractivos:

1. Cuevas de Punta del Este.

2. Laguna de Alvariño.

3. Faro de Carapachibey.

4. Poblado de Cocodrilo.

5. Parque Nacional Marino de Punta Francés.

6. Estero las Piedras.

7. Rincón del Guanal (Playa).

8. Guayacanal (Recurso Forestal).

9. Ensenada de la Siguanea.

10. Playa Larga.

11. Playa Blanca.

12. Playa de Punta del Este.

2.3.1- Valoración del estado y conservación de los recursos.

Una vez definidos los recursos y tractivos de la zona, se realizó a cada uno una valoración de su estado y conservación, su uso actual, las tendencias para su uso y el valor del potencial que tienen para su uso. Los resultados de este análisis se muestran en la siguiente tabla. 
Tabla 2. Valoración del estado conservatorio y de uso de los recursos.

\begin{tabular}{|c|c|c|c|c|c|}
\hline$\#$ & Recurso/ Atractivo & $\begin{array}{c}\text { Valor } \\
\text { Conservatorio }\end{array}$ & $\begin{array}{c}\text { Uso Actual } \\
\mathbf{y} \\
\text { Tendencia }\end{array}$ & $\begin{array}{c}\text { Uso } \\
\text { Potencial }\end{array}$ & $\begin{array}{c}\text { Limitaciones } \\
\text { para su uso }\end{array}$ \\
\hline 1 & $\begin{array}{c}\text { Cuevas de Punta del } \\
\text { Este }\end{array}$ & medio & bajo & alto & si \\
\hline 2 & Laguna de Alvariño & alto & bajo & alto & si \\
\hline 3 & $\begin{array}{c}\text { Faro de } \\
\text { Carapachibey }\end{array}$ & medio & bajo & medio & si \\
\hline 4 & $\begin{array}{l}\text { Poblado de } \\
\text { Cocodrilo }\end{array}$ & medio & medio & alto & si \\
\hline 5 & $\begin{array}{c}\text { Parque Nacional } \\
\text { Marino de Punta } \\
\text { Francés }\end{array}$ & medio & medio & alto & si \\
\hline 6 & Estero las Piedras & alto & bajo & medio & si \\
\hline 7 & Rincón del Guanal & alto & medio & alto & si \\
\hline 8 & Guayacanal & medio & bajo & alto & si \\
\hline 9 & $\begin{array}{l}\text { Ensenada de la } \\
\text { Siguanea }\end{array}$ & alto & medio & alto & si \\
\hline 10 & Playa Larga & alto & medio & alto & si \\
\hline 11 & Playa Blanca & alto & medio & alto & si \\
\hline 12 & $\begin{array}{c}\text { Playa de Punta del } \\
\text { Este }\end{array}$ & alto & medio & alto & si \\
\hline
\end{tabular}

Fuente: Elaboración propia.

Esta tabla demuestra que en sentido general estos recursos y atractivos presentan un nivel medio de conservación y esto se debe al bajo uso que se les da actualmente, lo que ha provocado que no se invierta adecuadamente en su conservación y/o restauración. No obstante, el valor patrimonial, folclórico, natural o único que estos poseen les otorga un alto potencial para su uso futuro.

Epígrafe 2.3. Evaluación Turística de los recursos y atractivos.

Teniendo en cuenta sus características y su alto nivel de atractividad, se seleccionaron para realizar su evaluación turística los siguientes recursos o atractivos a los cuales también se les realizó la ficha técnica de inventario (anexo 2): las Cuevas de Punta del Este, la Laguna de Alvariño y el Poblado de Cocodrilo. Se tuvieron en cuenta los criterios empleados por el Doctor Eros Salinas (2013) en su evaluación realizada a 5 lugares del Municipio Madruga. La escala de valores fue de 1 y 5 puntos, siendo el 1 el más bajo y 5 el más alto. El análisis de los resultados se realizó a partir de sumar los promedios de las evaluaciones individuales en cada uno de los aspectos. Los resultados se reflejaron en la siguiente tabla. 
Tabla 3. Evaluación turística.

\begin{tabular}{ccccc}
\hline Evaluación/sitio & $\begin{array}{c}\text { Cuevas } \\
\text { de Punta } \\
\text { del Este }\end{array}$ & $\begin{array}{c}\text { Laguna } \\
\text { de } \\
\text { Alvariño }\end{array}$ & $\begin{array}{c}\text { Poblado de } \\
\text { Cocodrilo }\end{array}$ & Total \\
\hline $\begin{array}{c}\text { Estética } \\
\text { Funcional }\end{array}$ & 3 & 5 & 4 & 12 \\
$\begin{array}{c}\text { Conservacionista } \\
\text { higiénico- }\end{array}$ & 5 & 5 & 4 & 13 \\
$\begin{array}{c}\text { sanitario } \\
\text { Técnico- }\end{array}$ & 2 & 5 & 3 & 13 \\
económico & 2 & 2 & 3 & 7 \\
Total & $\mathbf{1 8}$ & $\mathbf{2 1}$ & $\mathbf{1 8}$ & \\
\hline
\end{tabular}

Fuente: Elaboración Propia.

La mayor puntuación fue alcanzada por la Laguna de Alvariño, quedando con menos puntuación las Cuevas de Punta del Este y el Poblado de Cocodrilo. Pese a esto, estos no equidistan demasiado entre ellos, por lo que de manera general los 3 sitios poseen buenas condiciones para la realización de actividades turísticas.

Los aspectos mejor evaluados fueron el funcional, y el conservacionista, todos con 13 de 20 puntos posibles lo cual indica que de manera general los lugares cumplen con el objeto social que tienen creado y poseen un alto valor natural, cultural e histórico que hace que sea necesaria su conservación.

Por otra parte, con solo 7 puntos, el aspecto peor evaluado es el técnico económico, lo cual se debe a una incorrecta política inversionista y de uso de estos lugares para el desarrollo del turismo y además por la escasez de recursos monetarios con que cuenta el Municipio Especial en sentido general.

Al sumarse las puntuaciones totales de cada sitio para obtener una puntuación general que permitiera evaluar la zona seleccionada. El resultado alcanzado por la Zona Sur de la Isla de la Juventud fue de 57 puntos lo cual representa el $76 \%$ de la puntuación total (al sumarse 4 sitios la máxima puntuación posible es de 75 puntos) lo que indica que la zona tiene un alto potencial para el desarrollo de actividades turísticas por lo que es necesario realizar inversiones y proyectos que permitan un correcto empleo de los recursos y atractivos de la zona a fin de lograr un desarrollo sostenible del turismo en ella.

Epígrafe 2.4. Principales problemas del área de estudio.

Pese a que ya se ha demostrado el alto potencial que posee el Sur para el desarrollo de la actividad turística, es también cierto que esta es muy baja en la zona; lo que evidencia la existencia de dificultades para su gestión como posible destino turístico. Es por ello que se hace necesaria la implementación de acciones que permitan eliminar estas dificultades, pero, para que esto sea posible es necesario conocer cuáles son estas. 
Por estas razones se elaboró un banco con los principales problemas que presenta el Sur, los cuales fueron agrupados a criterio del autor en las áreas estratégicas, consideradas por este como las más importantes para el desarrollo turístico de la zona. Las mismas fueron: Infraestructura, Comercialización, Gestión y Recursos Humanos. Los resultados se exponen a continuación en la siguiente tabla:

Tabla 4. Banco de Problemas.

\section{Áreas/Problemas}

Infraestructura

Insuficiente infraestructura para la prestación de servicios

Acceso a los sitios

Carencia de infraestructura para las telecomunicaciones

Bajo volumen de inversión

Deterioro de la infraestructura existente

Comercialización

Inexistencia de una imagen sólida como destino

Deficiente empleo de las TIC para la comercialización de los sitios

Poca diversificación de la oferta

Alta dependencia del Crucerismo

Baja Atracción de Flujos Turísticos

Recursos Humanos

Insuficiente profesionalización del personal

Bajo dominio de lenguas extranjeras

Carencia de personal calificado para el guiaje

Carencia de personal calificado para la superación de la fuerza de trabajo

existente.

\section{Gestión}

Insuficiente uso de la ciencia y la técnica para el manejo del área

Poca claridad en la visión integrada del Sur como posible destino turístico

Implementación de modelos de gestión desactualizados

\section{Clasificación}

Muy Grave

Grave

Poco Grave

Muy Grave

Grave

Muy Grave

Grave

Poco Grave

Grave

Grave

Grave

Poco Grave

Grave

Poco Grave

Grave

Grave

Grave

\section{Fuente: Elaboración Propia.}

\section{Resultados.}

Objetivos por áreas.

De acuerdo con los análisis realizados en la investigación sobre las deficiencias y necesidades del Sur; se definieron los siguientes objetivos para las áreas estratégicas seleccionadas para el desarrollo de la actividad turística en la zona.

\section{Comercialización:}

- Consolidar la imagen del territorio.

- Realizar un mejor uso de las Tecnologías de la Información y las Comunicaciones. 
- Captar nuevos segmentos de demanda.

- Atraer mayor flujo turístico.

\section{Infraestructura:}

- Contar con la infraestructura necesaria para la realización de la actividad turística en el destino.

- Mejorar y mantener el estado de conservación de la infraestructura existente.

\section{Recursos Humanos:}

- Contar con un personal altamente calificado y preparado para satisfacer las necesidades de la demanda.

\section{Administración:}

- Realizar un manejo sostenible de la actividad turística en el territorio.

\section{Propuestas de acciones.}

Como ya se ha demostrado anteriormente, los sitios de estudio escogidos presentan características similares, por lo que se realizó una propuesta de acciones general. Para lograr el cumplimiento de los objetivos trazados para las áreas estratégicas se proponen las siguientes acciones:

- Difusión de la imagen del destino a través de las redes sociales y el empleo de sitios web.

- Diseñar estrategias de comercialización y marketing.

- Diversificar la oferta incrementando las actividades de Turismo Náutico, Deportivo, Ecoturismo, Turismo de Aventuras y Cultural.

- Establecer contacto y entablar relaciones con AA. VV y TT. OO especializados.

- Reinvertir las ganancias del territorio y captar nuevas inversiones para la creación de infraestructura.

- Incrementar la inversión extranjera.

- Aumentar la capacidad de alojamiento mediante la construcción de instalaciones ecológicas.

- Implementar instalaciones alternativas para el alojamiento (hoteles flotantes, campamentos ecológicos y estaciones biológicas).

- Construcción de instalaciones complementarias al alojamiento (instalaciones de restauración, recreación, y deportivas).

- Realizar labores de restauración y mantenimiento a las infraestructuras existentes.

- Mejorar las vías de acceso.

- Implementar planes de capacitación para el personal.

- Incentivar la participación de la población local dentro del personal.

- Actualizar los planes y modelos de gestión del territorio. 


\section{Conclusiones.}

En la presente investigación se arribaron a las siguientes conclusiones:

1. El Sur de la Isla de la Juventud es una de las áreas protegidas más importantes del país, declarada sitio RAMSAR, la cual cuenta con una flora y fauna excepcional y una población influenciada por la presencia del primer asentamiento de caimaneras en la Isla de la Juventud.

2. El Sur de la Isla de la Juventud posee recursos y atractivos turísticos que le otorgan grandes potencialidades para el desarrollo del turismo, pero no son aprovechados debido a la falta de infraestructura, la mala comercialización y la escaza inversión en el territorio.

3. Las principales acciones para fomentar el desarrollo turístico del Sur deben enfocarse principalmente en la construcción y perfeccionamiento de la infraestructura, la aplicación de una comercialización efectiva y la implementación de una gestión eficaz y eficiente en la zona, que permita el desarrollo de un turismo sostenible.

\section{Referencias Bibliográficas}

Álvarez, G. L. (12 de febrero de 2018). Especialista en Comercialización de la Agencia ECOTUR en la Isla de la Juventud. (D. C. Rodríguez, Entrevistador).

Boo, E. (1990). Ecoturismo: Potencialidades y Escollos. WWF., The Conservation Found. Washington DC. USA. 226 pp.

Ceballos - Lascurain, H. (1996). Tourism, Ecotourism and Proted Areas. IUCN, Suiza. 301 pp.

Crispin, D. Metodología para realizar caminatas ecoturísticas en la Isla de la Juventud (Cuba) como recreación sostenible. APUNTS. Educación Física y Deportes. 2016, \# 123, 1er trimestre (enero-marzo), Índex. ISSN-1577-4015. Pág. 86. Depósito Legal: B-16059-90.).

Crispin, D. Límites en la capacidad de carga de visitantes de Ecoturismo en Punta del Este, Isla de la Juventud (Cuba). Investigaciones Turísticas, (13). Enero - junio 2017. pp. 96-113. ISSN. 2174-5609. http://dx.doi.org/10.461/INTURI2017.13.05

Crispin, D. (18 de febrero de 2018). Profesor Titular de la Facultad de Cultura Física y Deporte de la Universidad de la Isla de la Juventud 'Jesús Montané Oropesa " y especialista en Ecoturismo. (D. C. Rodríguez, Entrevistador).

Empresa Nacional de Flora y Fauna. (2015). Plan de Manejo Área Protegida de Recursos Manejados Sur de la Isla de la Juventud Sitio RAMSAR. Isla de la Juventud.

Lecturas de Ecoturismo (1996) - Universidad de la Habana - Canadian Internacional development Agency - 106 p. 
Oficina Nacional de Estadística e Información. (2016). Anuario estadístico 2015 isla de la juventud. Nueva gerona.

Panorama OMT del Turismo Internacional Edición 2015 [en línea]. España: "Datos y Cifras" Organización Mundial del Turismo, (2016). [fecha de consulta: 22 octubre 2018]. Formato pdf. Disponible en: http://www.e-unwto.org/doi/book/10.18111/9789284416875 Wednesday, July 22, 2015 4:00:02 AM - IP Address: 173.243.43.210. Copyright () 2015, Organización Mundial del Turismo (OMT-UNWTO) Todos los derechos reservados.

Picornell, C. (2015). Los impactos del Turismo. Papers de Turisme, 11, 65-91. 


\section{Para citar el artículo indexado.}

Crispín Rodríguez, D., Malvarez Cuello, A., Espinosa González, C. A., \& López Efraín, V. (2019). Propuesta de acciones turísticas para el Sur de la Isla de la Juventud. Explorador Digital, 3(4), 113-131. https://doi.org/10.33262/exploradordigital.v3i4.938

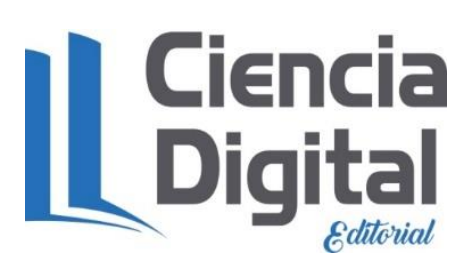

El artículo que se publica es de exclusiva responsabilidad de los autores y no necesariamente reflejan el pensamiento de la Revista Explorador Digital.

El articulo queda en propiedad de la revista y, por tanto, su publicación parcial y/o total en otro medio tiene que ser autorizado por el director o editor de la Revista Explorador Digital.

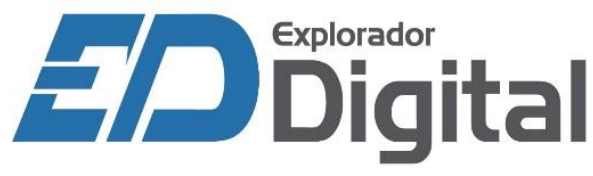

